\title{
Effect of electroacupuncture on chemotherapy-induced diarrhea: study protocol for a randomized controlled trial
}

\author{
Yuhang Wang ${ }^{1,2 \#, ~ M i n g f e i ~ Z h o u ~}{ }^{3 \#}$, Jianmei Shen ${ }^{4}$, Dongsheng Wang ${ }^{4}, \mathrm{Na} \mathrm{Xu}^{5}$, Yafang Song ${ }^{5}$, \\ Mengzhu Sun ${ }^{1,2}$, Lu Chen ${ }^{6}$, Lixia Pei ${ }^{6}$, Jianhua Sun $^{6}$ \\ ${ }^{1}$ Department of Acupuncture and Rehabilitation, Affiliated Hospital of Nanjing University of Chinese Medicine, Nanjing, China; ${ }^{2}$ Acupuncture \\ and Tuina Academy, Nanjing University of Chinese Medicine, Nanjing, China; ${ }^{3}$ Department of Oncology, Nanjing Xiaohang Hospital, Nanjing, \\ China; ${ }^{4}$ Department of Oncology, Nanjing Red Cross Hospital, Nanjing, China; ${ }^{5}$ Acupuncture and Tuina Academy, Nanjing University of Chinese \\ Medicine, Nanjing, China; ${ }^{6}$ Department of Acupuncture and Rehabilitation Province Hospital of Traditional Chinese Medicine, Affiliated Hospital \\ of Nanjing University of Traditional Chinese Medicine, Nanjing, China \\ Contributions: (I) Conception and design: Y Wang; (II) Administrative support: M Zhou, J Sun, L Pei; (III) Provision of study materials or patients: M \\ Zhou, J Shen, D Wang; (IV) Collection and assembly of data: N Xu, L Chen; (V) Data analysis and interpretation: Y Song, M Sun; (VI) Manuscript \\ writing: All authors; (VII) Final approval of manuscript: All authors. \\ "These authors contributed equally to this work. \\ Correspondence to: Jianhua Sun. Department of Acupuncture and Rehabilitation Province Hospital of Traditional Chinese Medicine, Affiliated \\ Hospital of Nanjing University of Traditional Chinese Medicine, Nanjing, China. Email: 377201634@qq.com.
}

Background: Chemotherapy-induced diarrhea (CID) is one of the side effects of chemotherapy. Diarrhea not only affects the overall treatment effectiveness but also reduces patients' quality of life. Severe diarrhea can lead to electrolyte imbalance and even be life-threatening. Although acupuncture has been widely used in clinical practice and its effectiveness for managing functional diarrhea has been recognized, there is no sound evidence of its efficacy in managing CID. Therefore, the aim of the proposed randomized controlled trial is to examine the effectiveness and potential risks of using acupuncture for the management of CID and to describe its protocol herein.

Methods: This trial will be conducted in a double-blinded manner and comprise two arms that will be investigated across multiple centers in parallel. The study cohort will comprise 168 outpatients who have CID from six Chinese hospitals. The patients will be randomly and equally divided between an intervention group (electroacupuncture) and a control group (micro-electroacupuncture). In the former, acupuncture will be performed with the conventional method to induce the de qi sensation, and in the latter group, acupuncture will be performed with a sham procedure that does not involve the insertion of needles. The acupoints ST25, SP14, SP6, and ST37 will be applied in the two methods. These procedures will be performed three times a week for four consecutive weeks. The number of days on which CID occurred, the incidence of CID, and fecal characteristics are considered as the primary outcomes, and the Functional Assessment of Chronic Illness Therapy for Patients with Diarrhea subscale score and the World Health Organization Quality of Life assessment are considered as secondary outcomes. The patients will be closely observed for complications and fluctuations in vital signs.

Discussion: If the findings from the trial demonstrate the effectiveness and safety of using acupuncture to treat CID, they could serve as evidence for the clinical application of acupuncture as a complementary treatment for cancer patients during chemotherapy.

Trial registration: Chinese Clinical Trial Registry, ChiCTR2000035715, registered on August 16, 2020.

Keywords: Chemotherapy-induced diarrhea (CID); cancer; acupuncture; randomized controlled trial

Submitted Dec 08, 2020. Accepted for publication Mar 26, 2021.

doi: $10.21037 /$ tcr-20-3400

View this article at: http://dx.doi.org/10.21037/tcr-20-3400 


\section{Introduction}

The incidence and death due to cancer have not been fundamentally reversed (1). Chemotherapy is a major cancer treatment (2), and chemotherapy-induced diarrhea (CID) is a common adverse reaction in cancer patients, with an incidence of $50-80 \%$ (3). CID not only adversely affects the health and quality of life of patients (4) but also leads to dehydration and electrolyte imbalance, decreases blood volume, increases the probability of infection, and even endangers life. Furthermore, it could lead to the interruption of chemotherapy and limit the smooth implementation of the chemotherapy regimen, thereby reducing treatment effectiveness (5). The incidence of grade 3 or 4 diarrhea in patients using fluoropyrimidines and irinotecan was $5-44 \%(6,7)$. Although the pathogenesis of CID is not completely clear, the possible causes include drug-induced damage to intestinal epithelium (8), intestinal infection (9), intestinal inflammation (10), and use of antibiotics (11-13).

The routine treatment of CID includes opioids, antidiarrheal drugs, and mucosal protective agents, such as loperamide or octreotide (14); however, the therapeutic effect is sometimes unsatisfactory. For example, loperamide does not effectively relieve abdominal colic and occasionally causes side effects such as loss of appetite, dysphagia, fatigue, chest pain, and gastrointestinal bleeding (15). Further, long-acting octreotide acetate is not effective in preventing or relieving diarrhea in cancer patients receiving chemotherapy (16). In short, an effective and safe clinical treatment for CID is lacking.

Studies have found that acupuncture can alleviate the symptoms of diarrhea by stimulating the expression of endogenous opioid peptides (17) and regulating intestinal nerve function $(18,19)$. Therefore, acupuncture can improve the symptoms of CID. It is important to use appropriate sham acupuncture and blind methods to conduct highquality randomized controlled trials (RCTs) of acupuncture and moxibustion for the treatment of CID. To this end, the present trial was proposed to examine the effectiveness and potential risks of using acupuncture for the management of CID. The findings from such a trial could shed light on the applicability of acupuncture as a therapeutic method for CID.

We present the following article in accordance with the SPIRIT reporting checklist (available at http://dx.doi. org/10.21037/tcr-20-3400).

\section{Methods}

\section{Objective}

This is a research protocol of a randomized controlled clinical trial about Electroacupuncture to relieve CID, in this study, the efficacy and safety of electroacupuncture in relieving CID will be evaluated.

\section{Trial design and setting}

This is a multicenter, assessor-blinded RCT that was designed in accordance with the guidelines of the Consolidated Standards of Reporting Trials Statement. This trial is expected to take place over 8 weeks: a 4-week acupuncture treatment and a 4-week follow-up phase. Based on the criteria for inclusion in the trial, 168 patients will be recruited from six hospitals and will be randomly and equally divided into an electroacupuncture group $(n=84)$ and a micro-electroacupuncture or control treatment group $(n=84)$. A diagram depicting the protocol for the study is presented in Figure 1. Further, Table 1 shows the time points for treatment sessions and recording of data.

\section{Recruitment}

The trial will be performed by recruiting outpatients at in the following six hospitals: Affiliated Hospital of Nanjing University of Chinese Medicine, Nanjing Red Cross Hospital, People's Hospital of Guangxi Zhuang Autonomous Region, Affiliated Hospital of Nantong University, Affiliated Hospital of Jiangnan University, and Nanjing Xiaohang Hospital. Information about the trial will be placed on the bulletin board of all the participating hospitals and universities, as well as public advertisement boards in the area. Further, online advertisements will also be placed on relevant sites.

\section{Inclusion criteria}

(I) Patients with a malignant tumor confirmed by pathology or cytology; (II) patients scheduled to receive or receiving platinum-based drug, paclitaxel, and vincristine treatment as planned, and having diarrhea after chemotherapy; (III) patients aged 18 to 75 years; (IV) patients with an expected survival period of $\geq 6$ months and Eastern Cooperative Oncology Group (ECOG) Performance Status of $\leq 3$; (V) patients without serious heart, liver, lung, or kidney disease; 


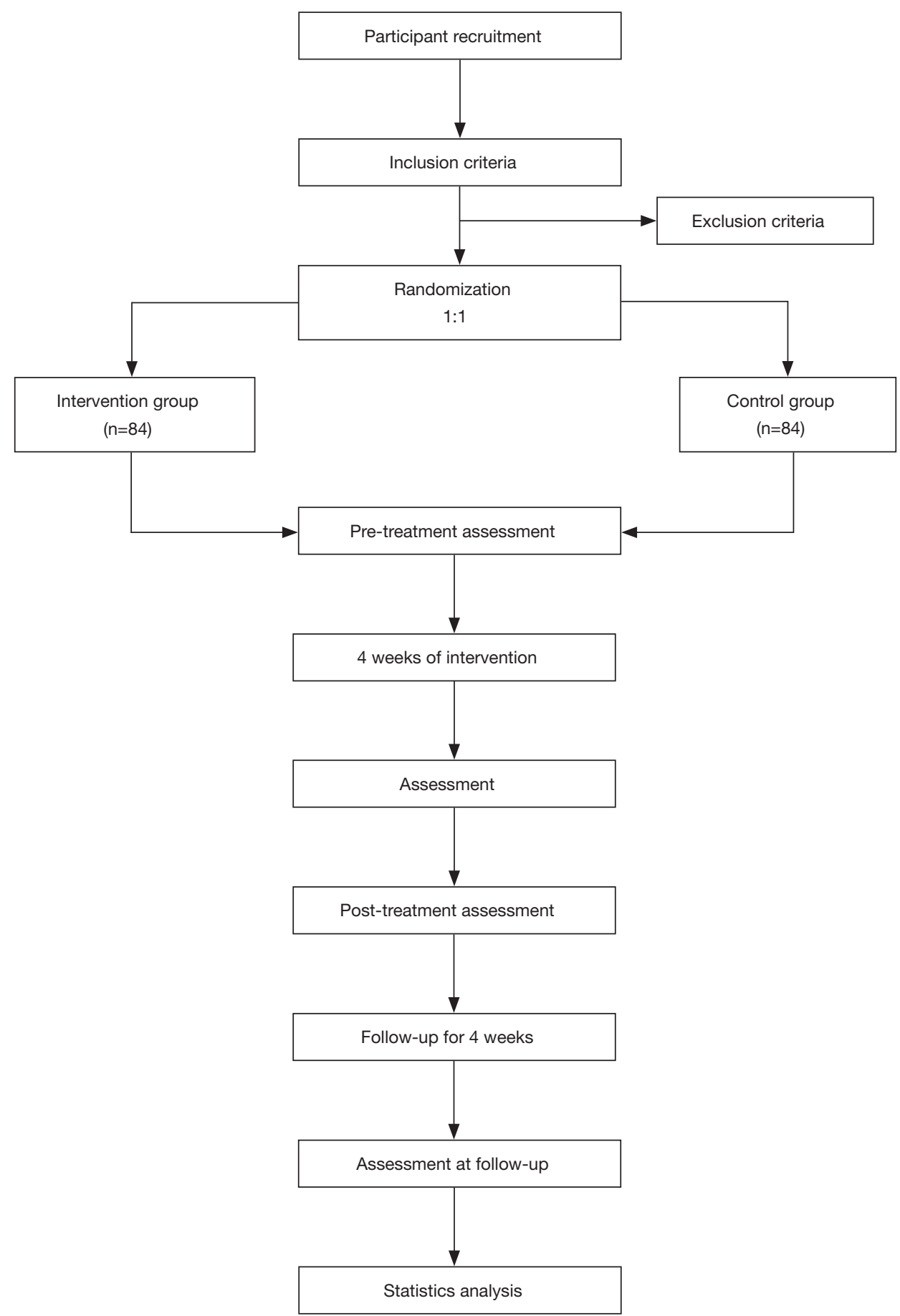

Figure 1 Participant flowchart showing the randomization of participants. Patients will receive 12 sessions of electroacupuncture treatment or sham electroacupuncture treatment lasting 30 minutes for 4 weeks, 3 times a week. 
Table 1 Timing of treatment visits and data collection

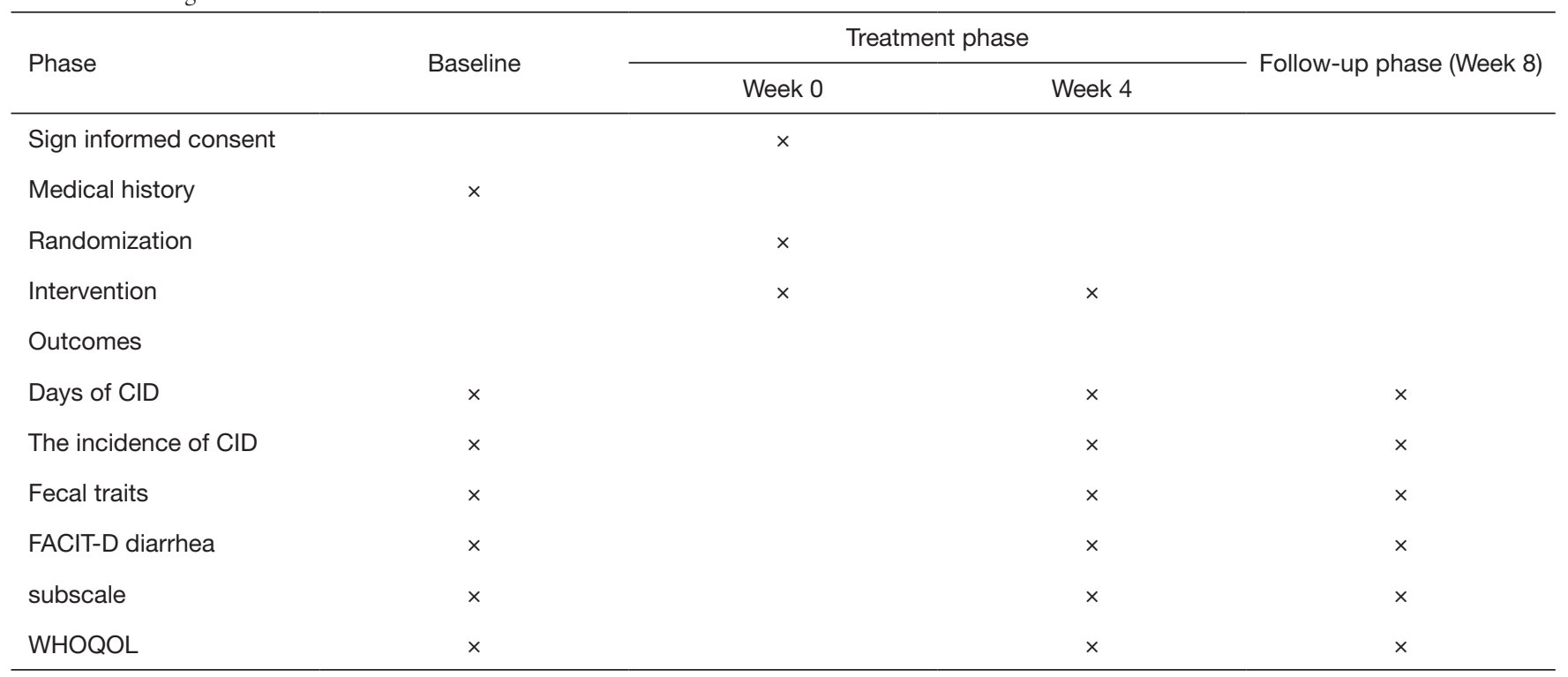

Table 1 shows the time for treatment sessions and recording of data. " $x$ " means information that must be collected and completed.

(VI) patients who voluntarily provided their informed consent in a written format and those who stated that they would abide by the instructions.

\section{Exclusion criteria}

(I) Upper respiratory tract infection and other symptoms of infection at the time of consultation; (II) use of antibiotics and other drugs that may cause diarrhea; (III) pregnant, breastfeeding, or planning to become pregnant during the trial period; (IV) electrocardiography abnormalities of clinical significance; (V) requirement of a pacemaker; (VI) gastrointestinal tumors that may affect bowel function as judged by a specialist; (VII) functional bowel disease or intestinal infectious disease; (VIII) colostomy; (IX) use of opioid analgesics; (X) severe intestinal obstruction and incomplete intestinal obstruction; (XI) severe coagulopathy and bleeding tendency; (XII) likelihood of severe gastrointestinal perforation; (XIII) mental illness; (XIV) receipt of last chemotherapy less than 4 weeks before study enrolment; (XV) lactose intolerance; (XVI) celiac disease; (XVII) type 1 diabetes or uncontrolled type 2 diabetes.

\section{Patient withdrawal criteria}

The patient withdrawal criteria are as follows: (I) occurrence of adverse reactions judged as warranting treatment discontinuation by the doctor; (II) progression of the primary disease; (III) treatment of the primary disease requiring the use of other drugs that may cause diarrhea; (IV) other critical illnesses during the study period warranting treatment discontinuation; (V) loss to follow-up.

\section{Randomized grouping of participants}

An independent administrator (independent graduate student) will design a randomization table for the participating hospitals with the help of the block randomization method and the SAS software (version 9.3; SAS Institute Inc., Cary, NC). The hospitals will receive sequentially numbered envelopes (which are opaque) containing random numbers that will be stored within a safe, double-locked storage unit or cabinet. When participants who meet the study criteria provide their informed consent, an envelope assigned to that individual will be handed to the physician. The envelops will be handed out in sequential order. Based on the number in the envelope, the participant will be allocated to either group.

\section{Blinding}

Based on the add-on design, participants and their treating physicians cannot be blinded, but the physician will not examine the primary and secondary outcomes. Instead, the assessor (an independent graduate student) will be blinded and will be required to not discuss the treatment 

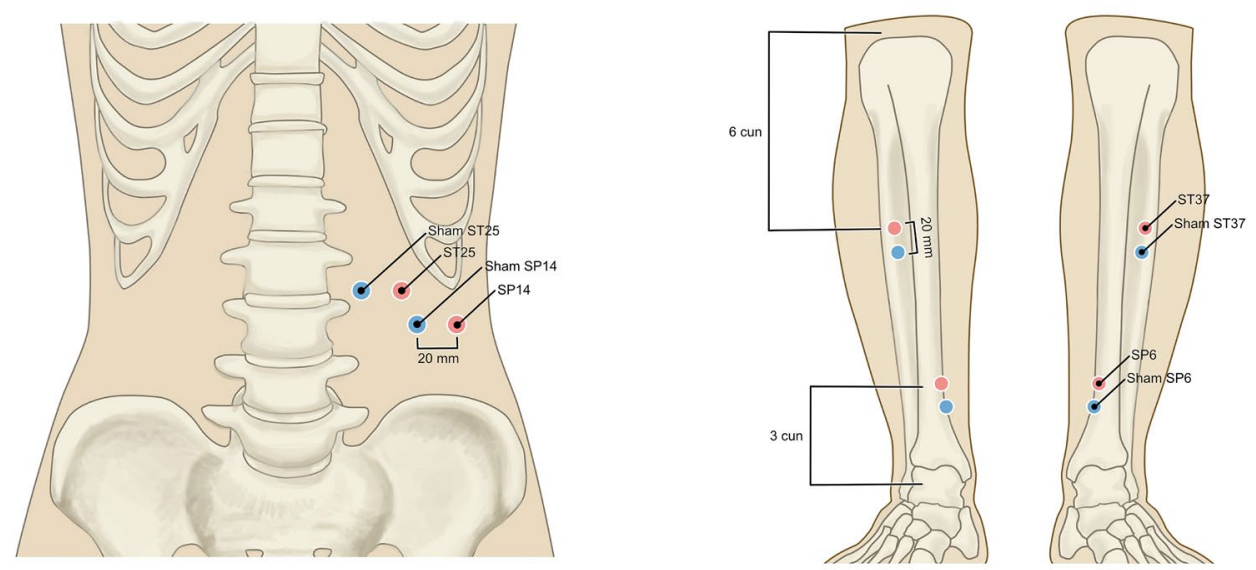

Figure 2 Location of acupoints for the electroacupuncture groups and sham electroacupuncture groups.

to maintain blinding. Additionally, the statistician (independent graduate student) and person collecting the data (an independent graduate student) will also be blinded. Unblinding can be performed at the end of the experiment for statistical analysis or when Serious adverse event (SAE) occurs during the research process. The unblinding will be carried out under the supervision of the researcher, doctor, and statistician (full-time graduate student). Subjects who are unblinded will no longer undergo clinical trials, and their data will not be included in the analysis.

\section{Intervention}

Disposable acupuncture needles $(0.3 \mathrm{~mm} \times 50 \mathrm{~mm}$ and $0.30 \mathrm{~mm} \times 75 \mathrm{~mm}$ in size), pragmatic placebo needles $(0.30 \mathrm{~mm} \times 25 \mathrm{~mm})$, and the SDZ-V device for electroacupuncture were obtained from Hua Tuo (Suzhou Medical Supplies Factory Co., Ltd., Suzhou, China). The participants will undergo their respective treatments separately in order to deter communication between them.

\section{The intervention group}

Participants in this group will receive real acupuncture treatment. Following disinfection of the skin area for treatment, sterile adhesive pads will be kept on the selected acupoints, that is, ST25, SP14, SP6, and ST37 (acupoint prescription based on consensus of acupuncture experts), on both sides, and the needles will be inserted via the pads approximately $15-50 \mathrm{~mm}$ into the skin. Following this step, the needles will be subjected to small and similar levels of twirling, lifting, and thrusting in order to induce de qi (which is defined as a combination of sensations that include soreness, numbness, distention, and heaviness, and is an important factor associated with the effectiveness of acupuncture) (20). For treatment delivery, a pair of electrodes will be attached across the needle handles at the four acupoints on both sides. A continuous stimulus will be delivered at a frequency of $10 \mathrm{~Hz}$ and a current of $0.5-2 \mathrm{~mA}$ : the stimulus is considered to be delivered if the skin in the area around the acupoints exhibit mild shivering that is not accompanied by pain. The participants will undergo 12 sessions in total over 4 consecutive weeks: three sessions will be conducted per week, each treatment lasts 30 minutes, with an optimal interval of 1 day between each session.

\section{The control group}

In the control group, a sham electroacupuncture procedure will be conducted with the pragmatic placebo needles. For the sham procedure, sham acupoints corresponding to the four treatment acupoints will be marked: the ST25 and SP14 sham points are $1 \mathrm{cun}(\approx 20 \mathrm{~mm})$ lateral to ST25 and SP14, and the sham SP6 and sham ST37 points are 1 cun $(\approx 20 \mathrm{~mm})$ vertical to SP6 and ST37 (Figure 2). The sham procedure will be conducted without insertion of the needle into the skin, all other treatment settings remain the same. Neither electrical stimulation nor hand manipulation induce De Qi (Figure 3).

\section{Outcome measures}

The primary outcomes will be examined at the baseline, in the fourth week after initiation of treatment, and in the eighth week of follow-up. All questionnaires will be in Chinese. 


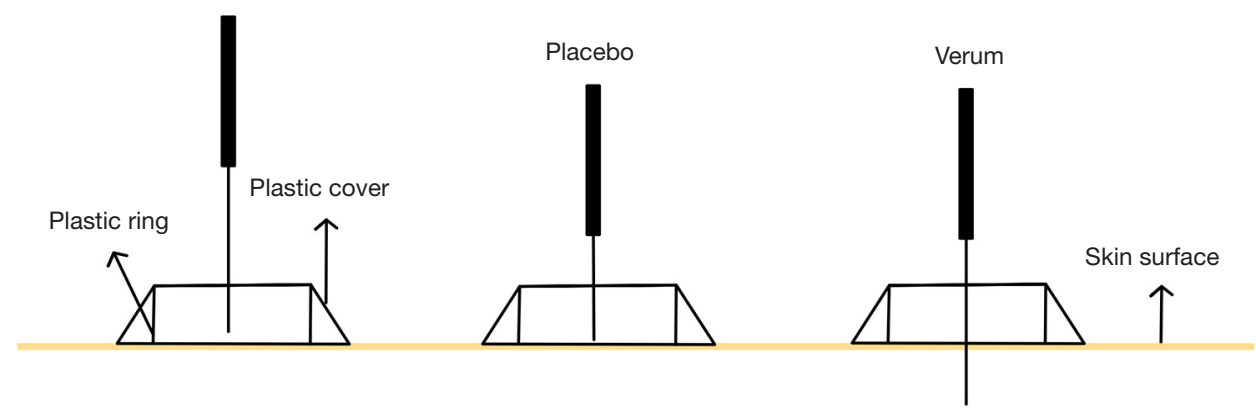

Figure 3 Schematic diagram of electroacupuncture and sham electroacupuncture device.

\section{Primary outcomes Days of CID}

Participants will be required to maintain a "defecation diary", and CID will be defined by number of stools $>1$ stools per day or more than the baseline level, as recorded in the "defecation diary" [Common Terminology Criteria for Adverse Events (CTCAE) Version 5.0]. The baseline level will be the average number of stools the subject recalls in the past 3 days (21).

The incidence of CID

This will be calculated by dividing the total number of occurrences of CID by the number of days that CID occurred.

\section{Fecal characteristics}

Participants will select the corresponding score according to the Bristol stool form scale, patient divides the total Bristol score of each stool in a day by the number of stools to get the average Bristol score of the day.

\section{Secondary outcomes}

Secondary outcomes are based on the FACIT-D diarrhea subscale and the WHOQOL, and will be assessed during the 8-week trial at the baseline, in the fourth week after initiation of treatment, and in the eighth week of follow-up. The questionnaires are in Chinese.

\section{Sample size}

The number of participants required to obtain statistical results of sufficient power will be determined via a sample size calculation method. For calculating this, the primary outcome is alteration in the duration of CID. In our previous pilot study, the change in the number of days of CID in the intervention group was shown to be $4.5 \pm 2.5$ $(\mathrm{n}=11)$ and that in the control group was $5.5 \pm 1.5 \quad(\mathrm{n}=11)$.
This is calculated based on a 2-tailed test, an allocation ratio of $1: 1$, a superiority trial, a power of $80 \%(1-\beta)$, and a significance level of $2.5 \%(\alpha)$. Further, under the assumption of a $20 \%$ dropout rate, the required sample size was calculated as 84 patients in each group or 168 patients in total.

\section{Safety assessment}

The participants are required to report any complications, adverse events, or related events associated with acupuncture treatment, and all the information will be recorded in detail in the Case Report Form. Potential adverse events include unfavorable or unexpected signs, symptoms, or diseases that may manifest over the course of the RCT. If an AE occurs due to participation in this study, the loss to the subject will be borne by the project team.

\section{Statistical analysis}

In order to reduce deviation, data will be assessed based on the intention-to-treat (ITT) principle. Measurement data will be analyzed with the $t$-test; ranked data, with the ranksum test; and categorical data, with the chi-squared test. The SPSS software (version 22.0; SPSS Inc., Chicago, IL, USA) will be used for all the analyses. The double-entry method will be used for data entry. Each branch center promptly submits material on paper to the project office, and the offline material and databases are maintained by the scientific research secretary.

The research team leader unit will establish a supervisory committee to regularly check the original data and system entry, and the experimental data will be accurately and diligently entered into the clinical trial public management platform ResMan. 


\section{Trial registration}

An application has been sent to the Chinese Clinical Trial Registry for the registration of this trial in order to ensure its transparency, high quality, and credibility.

\section{Patient and public involvement}

Six patient advisers from the six hospitals were included as co-applicants in the core study group, and they are expected to be present for all the teleconferences and meetings that are convened as part of this project. Additionally, a Public Involvement Panel comprising six to eight members with experience in acupuncture and tumor treatment will be set up at the Nanjing University of Chinese Medicine. The co-chair members of this panel will comprise the coapplicants. The role of the panel is to assist the core study group in various tasks, such as reviewing the information sheets and documents provided by the participants. The study protocol proposes four face-to-face meetings between all researchers and panel members and frequent updates and communication in the intervals between these meetings. The panel members will also be represented in the research advisory group as well as the Trial Steering Committee (TSC).

\section{Informed consent process}

The researcher will explain the entire contents of the informed consent form to potential participants. They will be given sufficient time and opportunity to ask about the details of the trial so that they can independently make an informed decision about participating in the research. The participants are required to write their name and date on the form, and in case of those who need an agent, the relationship with the agent must be clearly stated.

\section{Ethical statement}

The study was conducted in accordance with the Declaration of Helsinki (as revised in 2013). The study was approved by China Ethics Committee of Registering Clinical Trials (No. ChiECRCT20200160) and informed consent was taken from all the patients.

This study follows the principle of randomization, control, and double-blind. Through multiple centers, subjects were randomly divided into electroacupuncture group and control group at 1:1 to evaluate the effectiveness and safety of electroacupuncture in alleviating CID.

\section{Discussion}

The mechanisms underlying CID are yet to be completely elucidated, even though there is histopathological evidence that multiple pathways are involved in an imbalance in the absorptive and secretory processes of the small bowel (22). Diarrhea may be severe and, in some cases, associated with life-threatening dehydration and electrolyte abnormalities, but assessment and treatment of this condition are not currently standardized.

In China, acupuncture is a traditional treatment that has been in use for more than two millennia (23). The application of acupuncture has recently witnessed a revival in cancerrelated clinical research, where acupuncture is mainly used for cancer-related pain $(24,25)$, insomnia (26), psychological symptoms (27), fatigue (28), hot flashes (29), chemotherapyinduced peripheral neuropathy (30), breast cancer-related lymphedema (31), dyspnea in lung cancer (32), radiationinduced xerostomia (33) and other symptoms related to cancer. With regard to bowel function, acupuncture has shown potential in terms of ameliorating postoperative ileus (34) and gastroparesis syndrome in patients who undergo abdominal surgery (35). However, research on cancer-related diarrhea and constipation, both of which have been proven to be indications of acupuncture, are limited (36).

To ensure the quality of this RCT, the treating physicians will first undergo strict training on the appropriate technique for the sham procedure via several sessions conducted before the start of the study. Patients will be contacted on the phone to determine the appropriate treatment times in order to increase the attendance rate, and follow-up interviews will also be conducted by the researchers over the phone to collect and record information about treatment outcomes.

We have tried to standardize each step of the trial protocol, including selection of the acupoints, the acupuncture technique and steps, the needles and electroacupuncture apparatus, and the clinical experience of the physicians. We hope to relieve the symptoms of diarrhea after chemotherapy through electroacupuncture and reduce the incidence of frontal diarrhea in patients, improve patient's quality of life. Although there are some challenges in the process, we believe that this clinical trial will provide convincing evidence to support the use of acupuncture for the treatment of CID. 


\section{Trial status}

Participants are being recruited for the trail since July 15, 2020 , and the process will potentially be completed at the end of the year 2022 .

\section{Acknowledgments}

Funding: This work was supported by grants from the Leading Talents Project of Jiangsu Provincial Administration of TCM (grant no. SLJ0206) and the Nanjing University of Chinese Medicine's Jiangsu University Nursing Discipline Construction Funding Project (grant no. 2019YSHL062).

\section{Footnote}

Reporting Checklist: The authors have completed the SPIRIT reporting checklist. Available at http://dx.doi.org/10.21037/ tcr-20-3400

Peer Review File: Available at http://dx.doi.org/10.21037/tcr20-3400

Conflicts of Interest: All authors have completed the ICMJE uniform disclosure form (available at http://dx.doi. org/10.21037/tcr-20-3400). All authors report grants from Jiangsu Provincial Administration of TCM, grants from Nanjing University of Chinese Medicine, during the conduct of the study.

Ethical Statement: The authors are accountable for all aspects of the work in ensuring that questions related to the accuracy or integrity of any part of the work are appropriately investigated and resolved. The study was conducted in accordance with the Declaration of Helsinki (as revised in 2013). The study was approved by China Ethics Committee of Registering Clinical Trials (No. ChiECRCT20200160) and informed consent was taken from all the patients.

Open Access Statement: This is an Open Access article distributed in accordance with the Creative Commons Attribution-NonCommercial-NoDerivs 4.0 International License (CC BY-NC-ND 4.0), which permits the noncommercial replication and distribution of the article with the strict proviso that no changes or edits are made and the original work is properly cited (including links to both the formal publication through the relevant DOI and the license). See: https://creativecommons.org/licenses/by-nc-nd/4.0/.

\section{References}

1. Ma J, Jemal A, Fedewa SA, et al. The American Cancer Society 2035 challenge goal on cancer mortality reduction. CA Cancer J Clin 2019;69:351-62.

2. Perez-Herrero E, Fernandez-Medarde A. Advanced targeted therapies in cancer: Drug nanocarriers, the future of chemotherapy. Eur J Pharm Biopharm 2015;93:52-79.

3. Sharma R, Tobin P, Clarke SJ. Management of chemotherapy-induced nausea, vomiting, oral mucositis, and diarrhoea. Lancet Oncol 2005;6:93-102.

4. Mariotto AB, Yabroff KR, Shao Y, et al. Projections of the cost of cancer care in the United States: 2010-2020. J Natl Cancer Inst 2011;103:117-28.

5. Andreyev J, Ross P, Donnellan C, et al. Guidance on the management of diarrhoea during cancer chemotherapy. Lancet Oncol 2014;15:e447-60.

6. Benson $\mathrm{AB}$ 3rd, Ajani JA, Catalano RB, et al. Recommended guidelines for the treatment of cancer treatment-induced diarrhea. J Clin Oncol 2004;22:2918-26.

7. Kweekel D, Guchelaar HJ, Gelderblom H. Clinical and pharmacogenetic factors associated with irinotecan toxicity. Cancer Treat Rev 2008;34:656-69.

8. Kordes M, Gerling M. Variations in the management of diarrhoea induced by cancer therapy: results from an international, cross-sectional survey among European oncologists. ESMO Open 2019;4:e000607.

9. Lian Q, Xu J, Yan S, et al. Chemotherapy-induced intestinal inflammatory responses are mediated by exosome secretion of double-strand DNA via AIM2 inflammasome activation. Cell Res 2017;27:784-800.

10. Gibson RJ, Stringer AM. Chemotherapy-induced diarrhoea. Curr Opin Support Palliat Care 2009;3:31-5.

11. Ikuno N, Soda H, Watanabe M, et al. Irinotecan (CPT-11) and characteristic mucosal changes in the mouse ileum and cecum. J Natl Cancer Inst 1995;87:1876-83.

12. Osterlund P, Ruotsalainen T, Peuhkuri K, et al. Lactose intolerance associated with adjuvant 5 -fluorouracil-based chemotherapy for colorectal cancer. Clin Gastroenterol Hepatol 2004;2:696-703.

13. Abigerges D, Chabot GG, Armand JP, et al. Phase I and pharmacologic studies of the camptothecin analog irinotecan administered every 3 weeks in cancer patients. J Clin Oncol 1995;13:210-21. 
14. Gibson RJ, Keefe DM. Cancer chemotherapy-induced diarrhoea and constipation: mechanisms of damage and prevention strategies. Support Care Cancer 2006; 14:890-900.

15. Akel T, Bekheit S. Loperamide cardiotoxicity: "A Brief Review". Ann Noninvasive Electrocardiol 2018;23:e12505.

16. Zachariah B, Gwede CK, James J, et al. Octreotide acetate in prevention of chemoradiation-induced diarrhea in anorectal cancer: randomized RTOG trial 0315. J Natl Cancer Inst 2010;102:547-56.

17. Takahashi T. Acupuncture for functional gastrointestinal disorders. J Gastroenterol 2006;41:408-17.

18. Weng ZJ, Wu LY, Zhou CL, et al. Effect of electroacupuncture on $\mathrm{P} 2 \mathrm{X} 3$ receptor regulation in the peripheral and central nervous systems of rats with visceral pain caused by irritable bowel syndrome. Purinergic Signal 2015;11:321-9.

19. Shang HX, Wang AQ, Bao CH, et al. Moxibustion combined with acupuncture increases tight junction protein expression in Crohn's disease patients. World J Gastroenterol 2015;21:4986-96.

20. Zhou K, Fang J, Wang X, et al. Characterization of de qi with electroacupuncture at acupoints with different properties. J Altern Complement Med 2011;17:1007-13.

21. Perez D, Sharples KJ, Broom R, et al. A randomised phase IIb trial to assess the efficacy of ReCharge ice cream in preventing chemotherapy-induced diarrhoea. Support Care Cancer 2015;23:3307-15.

22. Kornblau S, Benson AB, Catalano R, et al. Management of cancer treatment-related diarrhea. Issues and therapeutic strategies. J Pain Symptom Manage 2000;19:118-29.

23. Yeung WF, Chung KF, Yung KP, et al. The use of conventional and complementary therapies for insomnia among Hong Kong Chinese: a telephone survey. Complement Ther Med 2014;22:894-902.

24. Deng G, Bao T, Mao JJ. Understanding the Benefits of Acupuncture Treatment for Cancer Pain Management. Oncology (Williston Park) 2018;32:310-6.

25. Hershman DL, Unger JM, Crew K. Acupuncture for Aromatase Inhibitor-Related Joint Pain Among Breast Cancer Patients. JAMA 2018;320:2270-1.

26. Garland SN, Xie SX, DuHamel K, et al. Acupuncture Versus Cognitive Behavioral Therapy for Insomnia in Cancer Survivors: A Randomized Clinical Trial. J Natl Cancer Inst 2019;111:1323-31.

27. Haddad NE, Palesh O. Acupuncture in the treatment of cancer-related psychological symptoms. Integr Cancer Ther 2014;13:371-85.

28. Wang Z, Li S, Wu L, et al. Effect of acupuncture on lung cancer-related fatigue: study protocol for a multi-center randomized controlled trial. Trials 2019;20:625.

29. Liu W, Qdaisat A, Lopez G, et al. Acupuncture for Hot Flashes in Cancer Patients: Clinical Characteristics and Traditional Chinese Medicine Diagnosis as Predictors of Treatment Response. Integr Cancer Ther 2019;18:1534735419848494.

30. Bao T, Seidman AD, Piulson L, et al. A phase IIA trial of acupuncture to reduce chemotherapy-induced peripheral neuropathy severity during neoadjuvant or adjuvant weekly paclitaxel chemotherapy in breast cancer patients. Eur J Cancer 2018;101:12-9.

31. Hou W, Pei L, Song Y, et al. Acupuncture therapy for breast cancer-related lymphedema: A systematic review and metaanalysis. J Obstet Gynaecol Res 2019;45:2307-17.

32. Bauml J, Haas A, Simone CB, 2nd, et al. Acupuncture for Dyspnea in Lung Cancer: Results of a Feasibility Trial. Integr Cancer Ther 2016;15:326-32.

33. Simcock R, Fallowfield L, Monson K, et al. ARIX: a randomised trial of acupuncture $\mathrm{v}$ oral care sessions in patients with chronic xerostomia following treatment of head and neck cancer. Ann Oncol 2013;24:776-83.

34. Meng ZQ, Garcia MK, Chiang JS, et al. Electroacupuncture to prevent prolonged postoperative ileus: a randomized clinical trial. World J Gastroenterol 2010;16:104-11.

35. Sun BM, Luo M, Wu SB, et al. Acupuncture versus metoclopramide in treatment of postoperative gastroparesis syndrome in abdominal surgical patients: a randomized controlled trial. Zhong Xi Yi Jie He Xue Bao 2010;8:641-4.

36. Zheng H, Li Y, Zhang W, et al. Electroacupuncture for patients with diarrhea-predominant irritable bowel syndrome or functional diarrhea: A randomized controlled trial. Medicine (Baltimore) 2016;95:e3884.

Cite this article as: Wang Y, Zhou M, Shen J, Wang D, Xu N, Song Y, Sun M, Chen L, Pei L, Sun J. Effect of electroacupuncture on chemotherapy-induced diarrhea: study protocol for a randomized controlled trial. Transl Cancer Res 2021;10(5):2516-2524. doi: 10.21037/tcr-20-3400 